\title{
General anaesthesia or sedation for percutaneous aortic valve implantation? The questionnaire results and authors' experience
}

\author{
Ewa M. Kucewicz-Czech ${ }^{1}$, Leszek Machej ${ }^{1}$, Kazimierz Kiermasz ${ }^{1}$, Andrzej Węglarzy ${ }^{1}$, Maria Damps ${ }^{2}$, \\ Damian Hudziak ${ }^{3}$, Radosław Gocoł ${ }^{3}$, Andrzej Ochała ${ }^{4}$, Radosław Parma ${ }^{4}$ \\ ${ }^{1}$ Department of Cardiac Anaesthesia and Intensive Care Leszek Giec Upper-Silesian Medical Centre, Medical University \\ of Silesia, Katowice, Poland \\ 2Department of Anaesthesiology and Intensive Care, Upper Silesian Child Health Centre, Katowice, Poland \\ ${ }^{3}$ Department of Cardiac Surgery, Leszek Giec Upper-Silesian Medical Centre, Medical University of Silesia, Katowice, Poland \\ ${ }^{4}$ Third Department of Cardiology, Leszek Giec Upper-Silesian Medical Centre, Medical University of Silesia, Katowice, Poland
}

Kardiochir Torakochir Pol 2020; 17 (4): 198-202

\begin{abstract}
Introduction: Over the last two decades transcatheter aortic valve replacement (TAVR) has been approved for clinical use. The anaesthetic choice for this procedure is evolving. General anaesthesia was the predominant anaesthetic technique. Growing experience and advances in technology and economic considerations have led to an increasing interest in performing TAVR under monitored sedation.

Aim: The assessment of monitored sedation, called cooperative sedation, involves pharmacologically mediated suppression of consciousness and preservation of verbal contact in response to stimulation as a safe method of anaesthesia for TAVR.

Material and methods: Sixty out of 63 TAVR patients with femoral access received monitored sedation. Dexmedetomidine was administered in most of such cases (46 patients). A questionnaire was also carried out by staff involved in performing TAVR procedures, with more than 5 years of experience in it, concerning the method of anaesthesia and perioperative care.

Results: Conversion to general anaesthesia was required in $10 \%$ of patients (6 cases), only one as a patient-related complication (hypercarbia). The questionnaire carried out showed that anaesthesia and postoperative care after TAVR are underestimated. Conclusions: The preliminary results regarding anaesthetic management in TAVR procedures demonstrate that monitored sedation is safe, provided that contraindications are observed.
\end{abstract}

Key words: transcatheter aortic valve replacement, anaesthesia, conversion.

\section{Introduction}

Demographic data are unquestionable. In the United States, 2.5 million inhabitants over the age of 75 have been diagnosed with aortic valve stenosis. Since the number of individuals aged $>75$ years will double by 2050, the number of diagnosed aortic valve stenosis cases is going to increase significantly [1]. For many decades, the gold standard for management of aortic valve stenosis was surgical aortic valve replacement (SAVR). Patients ineligible for surgical treatment were offered pharmacotherapy or balloon valvuloplasty [2]. During the last two decades transcatheter aortic valve replacement (TAVR) was introduced into clinical practice. The successful pioneering procedure was performed by Alain Cribier in 2002 [3]. Initially, implantations were performed in patients disqualified for surgical treatment. Over time, the indications were widened and included lower-risk patients. Moreover, the valve technology evolved. The catheter's diameter was reduced from $24 \mathrm{~F}$ (2002) to $14 \mathrm{~F}$ (2013) and the valve characteristics were adjusted. The PARTNER study findings published in 2013 concluded the first stage of research on TAVR [4], demonstrating that the outcomes of surgical treatment and transcatheter aortic valve implantation in high-risk patients with severe valve stenosis and dysfunction of the left ventricle are comparable. According to the authors, it is essential to carry out research to improve the valve construction, so that the number of perivalvular leakages and central nervous system (CNS) strokes can be limited [5]. Further studies have shown the comparable mortality rates in both methods of valve implantation and lower incidences of life-threatening complications in TAVR, which resulted in widening the indications for the use of this technique in

Address for correspondence: Prof. Ewa M. Kucewicz-Czech, Department of Cardiac Anaesthesia and Intensive Care Leszek Giec Upper-Silesian Medical Centre, Medical University of Silesia, Katowice, Poland, phone: +48604451 880, e-mail: j.ciesla@sccs.pl

Received: 26.06.2020, accepted: 24.10.2020. 
low- and medium-risk patients [6]. The most dangerous life-threatening TAVR complications include acute kidney failure, myocardial infarction and vascular complications requiring transfusions of red blood cells [7]. However, the enthusiasm for the transcatheter method in low- and medium-risk patients was damped by the PARTNER II study results [8]. At present, there are no comparisons of homogeneous groups regarding the two techniques of implantation in question. The authors provocatively encourage cardiac surgeons to apply modern, less invasive surgery, which can be competitive for transcatheter methods, and to abandon old-fashioned sternotomy. Additionally, the degenerative changes quickly progressing on transvascularly implanted valves are worth stressing.

The 2016 estimates reveal that in our country the number of candidates for transcatheter aortic valve implantation increases by 1220 a year [2].

The preferable vascular access for TAVR is the femoral artery route; when another access is necessary, the subclavian, carotid, transapical or interventricular septal approaches are available. The femoral route is considered the least invasive approach; therefore, it is used most often. The transapical procedure requires left anterior thoracotomy and is performed under general anaesthesia.

Implantations are conducted in a hybrid operating room, which enables X-ray imaging ( $\operatorname{arm} \mathrm{C}$ ), is equipped with interventional cardiology devices as well as extracorporeal circulation apparatus, and where any type of anaesthesia and advanced haemodynamic monitoring are feasible. The recommendations include direct pressure measurements in the left radial artery, insertion of a cannula to the right internal jugular vein and a sheath to pass an endocavitary electrode or a Swan-Ganz catheter, urinary catheterisation, and capnometry [9]. In carotid approaches, cerebral oximetry (near infrared spectroscopy - NIRS) is advised. According to the recommendations, the implantation team should include an interventional cardiologist, cardiac surgeon and cardiac anaesthesiologist [1].

The issue of optimal anaesthesia for transcatheter aortic valve implantation continues to be discussed. Three methods are considered, i.e. local anaesthesia, monitored sedation and general anaesthesia.

\section{Aim}

The aims of the article were to survey cardiologists', cardiac surgeons' and anaesthetists' opinions about anaesthetic care during the TAVR procedure on the basis of the prepared questionnaire and to assess preliminary experience with TAVR procedures (femoral access) performed under monitored sedation

\section{Material and methods \\ Questionnaire findings}

Preparing for the $6^{\text {th }}$ Conference of Percutaneous Treatment of Structural Heart Diseases, held in Katowice in March 2019, the authors carried out a questionnaire in five centres taking part in the conference. The questionnaire questions were answered by anaesthesiologists. The results are presented in Table I.

During the conference another questionnaire survey was conducted among participants of the TAVR anaesthetic management session. Analysis involved the responses of 18 physicians, 17 men, 8 anaesthesiologists, 5 cardiac surgeons and 5 cardiologists; 16 respondents had more than 5 years of TAVR experience.

Table I. Results of the questionnaire carried out among anaesthesiologists cooperating with 5 cardiac surgery departments regarding standard management of patients qualified for transcatheter aortic valve replacement

\begin{tabular}{|c|c|}
\hline Question & Response \\
\hline Year of the first TAVR procedure & $2008-2011$ \\
\hline Number of TAVRs performed in 2019 & 73-100/centre \\
\hline Total number of TAVRs & 177-500/centre \\
\hline Is anaesthesia for TAVR provided by a cardiac anaesthesiologist? & $\begin{array}{c}\text { Only in } 1 \text { centre - general anaesthesiologist; in the remaining } \\
\text { centres - cardiac anaesthesiologist }\end{array}$ \\
\hline Number of anaesthesiologists in the TAVR team & Only in 1 centre - the dedicated group \\
\hline Is the anaesthesia protocol for TAVR available at the department? & No \\
\hline Premedication & All centres - benzodiazepines \\
\hline Intraoperative monitoring & 1 centre - without deep vein catheterisation \\
\hline Participation in TAVR training & 1 centre - no \\
\hline Where is the hybrid operating room located? & 1 centre - outside the operating suite \\
\hline Is an anaesthesiologist a member of the Heart Team? & No \\
\hline $\begin{array}{l}\text { When does the anaesthesiologist visit the patient for the first } \\
\text { time? }\end{array}$ & One day prior to implantation \\
\hline What scale is used to evaluate the surgical risk? & EUROSCORE/ASA \\
\hline General anaesthesia: sedation ratio/conversions/complications & Only 1 centre - incomplete data; the remaining centres - no data \\
\hline
\end{tabular}


Table II. Analysis of anaesthetic management in transcatheter aortic valve replacement procedures performed between 14.03.2019 and 19.12.2019

\begin{tabular}{|c|c|}
\hline Parameter & Data \\
\hline \multirow{4}{*}{$\begin{array}{l}\text { Number of TAVR } \\
\text { procedures }\end{array}$} & 63 - transfemoral access \\
\hline & $14-$ transcarotid access \\
\hline & $1-$ transapical \\
\hline & $78-$ in total \\
\hline \multirow{2}{*}{$\begin{array}{l}\text { Kind } \\
\text { of anaesthesia }\end{array}$} & 18 - general anaesthesia with intubation/LMA \\
\hline & $60-$ monitored sedation \\
\hline \multirow{2}{*}{$\begin{array}{l}\text { TAVR - transcarotid } \\
\text { access }\end{array}$} & 5 - general anaesthesia with LMA \\
\hline & 9 - general anaesthesia with intubation \\
\hline \multirow[t]{2}{*}{$\begin{array}{l}\text { TAVR transfemoral } \\
\text { access }\end{array}$} & $\begin{array}{l}60 \text { - monitored sedation } \\
\text { (46-dexmedetomidine) }\end{array}$ \\
\hline & 3 - general anaesthesia with intubation (5\%) \\
\hline \multirow{4}{*}{$\begin{array}{l}\text { Conversion } \\
\text { to general } \\
\text { anaesthesia }\end{array}$} & 3 - procedure-related complications \\
\hline & 2 -vascular complications \\
\hline & $1-$ patient-related complications (hypercapnia) \\
\hline & $6-$ in total \\
\hline $\begin{array}{l}\text { Death after } \\
\text { conversion } \\
\text { (procedure-related } \\
\text { complication) }\end{array}$ & 1 - shock, ischaemia of abdominal organs \\
\hline
\end{tabular}

\section{Preliminary analysis of our experience with monitored sedation}

The preliminary analysis of TAVR anaesthetic procedures in the Upper-Silesian Cardiology Centre in KatowiceOchojec performed between 14.03.2019 and 19.12.2019, i.e. after the conference with the involvement of anaesthesiologists, demonstrates the revolutionary changes in the attitudes toward anaesthesia. Previously, a significant majority of patients was anaesthetized generally; moreover, when sedation was decided, the LMAs were used in almost all cases. Sedation was carried out using the continuous infusion of propofol. The outcomes of anaesthetic management in TAVR procedures performed during the last 10 months of 2019 are presented in Table II.

After in-depth literature analysis and discussion on TAVR, since March 2019 the attitude towards anaesthetic management in this procedure has changed.

Ninety-five percent of femoral access procedures were performed under monitored sedation. Premedication should not be administered. In most such cases (46 patients) dexmedetomidine was administered. This drug is recommended by the American Society of Anesthesiologists due to its action limiting the risk of delirium. Sedation was induced with an infusion of dexmedetomidine in a dose of $1 \mu \mathrm{g} / \mathrm{kg}$ for 15 minutes followed by an infusion in a dose of $0.2-0.7 \mu \mathrm{g} / \mathrm{kg} / \mathrm{h}$. Ketamine in a dose of $50-100 \mathrm{mg}$ was administered for some patients at the anaesthetist's discretion. The mean age in the group analysed was 80 years (min. 58; max. 93), which shows that the risk of delirium among these patients is very high.

\section{Results}

The findings of the questionnaire carried out among Polish specialists are listed in Table III. The number of physicians taking part in the debate is likely to evidence that the effects of anaesthesia and postoperative care on final outcomes are underestimated.

In the period discussed, conversion from monitored sedation to general anaesthesia was required in $10 \%$ of patients (6 cases). There were complications with three patients because of procedure-related complications, two patients because of vascular complications and only one on the account of a patient-related complication. This conversion, which resulted from clinical instability of the patient (hypercapnia), did not affect the outcome. One of these patients died on the next post-procedure day due to ischaemia of abdominal organs. The mean procedure time ranged from 1.5 to 2 hours. Patients under dexmedetomidine sedation in doses proposed in the text slept deeply enough not to have discomfort during induced VT.

\section{Discussion}

Local anaesthesia, deprived of basic patient comfort, does not aspire to be the optimal choice; financially, however, this method is a leader in the classification of anaesthetic procedures. During the $32^{\text {nd }}$ Congress of EACTA in Berlin held in 2017, American anaesthesiologists presented their opinions based on telephone conversations with patients several weeks after implantation. The patients anaesthetised only locally were strongly against such a procedure. They still vividly remembered the horror they experienced associated with a narrow, uncomfortable operating table, inability to change positions, numbness, and

Table III. The results of the questionnaire carried out among participants of the session on transcatheter aortic valve replacement anaesthesia held during the $6^{\text {th }}$ Conference of Percutaneous Treatment of Structural Heart Diseases, Katowice 2019

\begin{tabular}{lr} 
Question & $\begin{array}{c}\text { Response } \\
\text { Should an anaesthesiologist be a member of the heart team? }\end{array}$ \\
\hline Who should decide about the kind of anaesthesia? & $67 \%$ yes team decision during qualification \\
\hline Who should anaesthetise/supervise sedation? & $91 \%$ cardiac anaesthesiologist; $17 \%$ any specialist in anaesthesiology; \\
\hline Does anaesthesia affect treatment outcomes? & $56 \%$ anaesthetic nurse \\
\hline $\begin{array}{l}\text { Do meetings on and undoubtedly yes; } 39 \% \text { no; } 6 \% \text { I have no opinion } \\
\text { towards the procedure? }\end{array}$
\end{tabular}


pain. The two remaining methods of anaesthesia have their assets and drawbacks.

The assets of general anaesthesia include the prevention of pulmonary aspiration of gastric contents, monitoring of respiration, prevention of hypoxia and hypercapnia, feasibility of transoesophageal echocardiography (recommended for infective endocarditis (IE) and coexistence of the subvalvular membrane). General anaesthesia ensures excellent conditions in the operating field, controlled apnoea, and immobility. Moreover, the valve can be optimally positioned. The drawbacks of this method are associated with more common use of vasoactive drugs, increased incidences of infectious lung complications and acute kidney failure, longer hospitalisations and higher costs [10, 11]. A high risk of contrast-induced acute kidney failure is more common in patients with chronic kidney disease, diabetes mellitus and heart insufficiency [1]. Delayed mobilisation regards only the patients with vascular complications at the catheter insertion site. The drugs used for general anaesthesia include etomidate in a dose of $0.2-0.6 \mathrm{mg} / \mathrm{kg}$; rocuronium or vecuronium or cisatracurium; fentanyl in fractionated doses, $25 \mu \mathrm{g}$ each, or remifentanil infusions and propofol infusions or volatile sevoflurane [12].

Monitored sedation, called cooperative sedation, involves pharmacologically mediated suppression of consciousness and preservation of verbal contact in response to stimulation [11]. The advantages of this method are continuous contact with the patient, which means continuous monitoring of the central nervous system (CNS), haemodynamic stability, less frequent use of vasoactive drugs, quicker mobilisation, shorter hospitalization, and improved quality of life already 3 days after implantation. The disadvantages of this method include no control of patent airways, higher incidences of hypoxia and hypercapnia, uncontrolled movements during implantation, unpleasant sensations associated with quick rhythm stimulation, higher numbers of perivalvular leakages, and necessity to implant a stimulator [1]. Sedation can be performed with loading dose of dexmedetomidine, followed by an infusion. Propofol in fractionated $25 \mu \mathrm{g}$ each could be given. In our preliminary study, some patients were additionally given ketamine in a bolus (50-100 mg) as needed. Another pharmacological option is an infusion of remifentanil in a dose of $0.02-0.04 \mu \mathrm{g} / \mathrm{kg} / \mathrm{min}$ together with an infusion of propofol or ketamine [12]. When administering successive doses of fentanyl, an increasingly high risk of respiratory depression should be considered. Midazolam is not recommended since its time of action exceeds the time of a procedure and additionally increases the risk of delirium. Irrespective of the type of anaesthesia, premedication should not be administered.

Critical events occur even in the centres where numerous TAVR procedures are performed. According to analysis of Mayr et al., critical complications are observed in $9 \%$ of patients [13]. The authors have demonstrated that every second patient undergoing implantation requires infusions of vasoactive drugs; additionally, the mortality dur- ing procedures once complications have occurred is $0.3 \%$. According to them, such a good outcome is attributable to quick and adequate reactions of cardiac anaesthesiologists involved in the procedure. However, their opinion has not been confirmed by scientific evidence. Considering the present advanced level of TAVR, the presence of a cardiac anaesthesiologist during the procedure is the optimal solution. This theory was confirmed by our questionnaire. When the term "heart team" was entered into PubMed, 10355 positions were displayed. We reviewed some of them and found that the anaesthesiologist is an element of this structure.

When any complications occur, which are usually extremely severe, invasive hemodynamic monitoring has to be implemented and interpreted, decisions quickly taken and executed [14].

When monitored sedation is decided, we should realize that urgent conversion to general anaesthesia is an extremely severe complication, whose incidence is $17 \%$ (3.4-20\%). The available literature data indicate that this incidence tends to decrease once more experience is gained. Nevertheless, the fact is that urgent conversion results in $66 \%$ mortality; in $50 \%$ the above is caused by the lack of patient's cooperation (anxiety, back pain, dyspnoea in the dorsal decubitus position; in the remaining $50 \%$ it is caused by severe-procedure-related complications (aortic haemorrhage, tamponade) [15]. In our preliminary study, the conversion from monitored sedation to general anaesthesia was required in $10 \%$ of patients, in which one patient died due to complications of shock the following day.

The incidence rate of postoperative delirium, diagnosed in patients undergoing cardiac surgery, ranges between $8 \%$ and $66 \%$. Delirium not only prolongs hospitalizations but also increases the risk of death. Its incidence increases with patients' age. There are no differences in the incidences of delirium between patients undergoing TAVR and SAVR procedures [16]. In contrast, the incidence of this complication is significantly lower in patients with implantations through the femoral access route, as compared to those with implantations through any other access, including surgical. In $40 \%$ of patients, delirium can be avoided by modifying the risk factors of delirium. For instance, intraoperative neuromonitoring (cerebral oximetry) and monitoring of the depth of anaesthesia (bispectral index) are suggested [17]. Pharmacology used during anaesthesia is undoubtedly relevant for limiting the delirium risk. The drug of choice in prevention of delirium is dexmedetomidine [16]. Due to that dexmedetomidine was chosen in our study.

Regional paravertebral anaesthesia at the T3-T5 level in patients qualified for transapical implantation of the aortic valve enables reduction of the dose of opioids during the procedure and earlier extubation. In this group with additional local anaesthesia, the incidence of atrial fibrillation was lower [18]. Additional local anaesthesia is worth considering because the postoperative period should be focused on early extubation, quick mobilization and short hospitalization [1]. 
The methods of anaesthesia described above have their advantages and disadvantages, and none of them can be called optimal. The 2016 data demonstrate that in the United States, TAVRs under sedation are increasingly common; nevertheless, more than $80 \%$ of patients are still subjected to general anaesthesia. In Europe, the situation is different; $90 \%$ of patients undergo cooperative sedation. The exception is the UK, where all patients are subjected to general anaesthesia [1]. The most experienced centres recommend both options of intraoperative management - cooperative sedation and general anaesthesia [19]. The contraindications for sedation include lack of patient's consent, difficult airways, pulmonary hypertension, morbid obesity, chronic oxygen therapy, fragility, alcohol abuse, cognitive and neurodegenerative disorders, psychiatric diseases, chronic use of sedatives or antidepressants [1]. Summing up, general anaesthesia should be preferred for respiratory reasons (difficult intubation, sleep apnoea, high risk of aspiration), diseases in which patients cannot assume dorsal decubitus (back pain, diseases of joints and muscles, orthopnoea), hindered cooperation (procedure-related anxiety, dementia, cognitive disorders) and the surgical technique (alternative transvascular access) [15].

Another piece of evidence confirming the lack of an ideal method of anaesthesia for TAVR has been presented by specialists from the University Hospital in North Carolina, who introduced the protocol of early recovery after surgery (ERAS) to the procedure in question and, having considered all pros and cons, chose general anaesthesia [20]. They confirmed that the aim of anaesthetic management is early recovery, prevention of delirium and organ stability. They use propofol 50-100 $\mu \mathrm{g} / \mathrm{kg} / \mathrm{min}$ and remifentanil 0.05-0.08 $\mu \mathrm{g} / \mathrm{kg} / \mathrm{min}$, avoiding the administration of benzodiazepines and minimizing the dose of opioids.

\section{Conclusions}

The preliminary results regarding anaesthetic management in TAVR procedures demonstrate that monitored sedation is safe, provided that contraindications are observed. However, the presence of a cardiac anaesthesiologist during the procedure is strongly recommended, confirmed by Polish experts in this specific questionnaire.

\section{Disclosure}

The authors report no conflict of interest.

\section{References}

1. Goeddel LE. Abernathy JH, Brady MB. An Anesthesiologist Guide to the 2017 American College of cardiology Expert Consensus Decision Pathway for Transcatheter Aortic valve Replacement in the management of Adults with Aortic Stenosis. J Cardiothorac Vasc Anesthesia 2019; 33: 263-273.

2. Hamm C, Arsalan M, Mack MJ. The future of transcatheter aortic velve implantation. Eur Heart J 2016; 37: 803-810.

3. Cribier A, Eltchaninoff H, Bash A, Borenstein N, Tron C, Bauer F, Derumeaux G, Anselme F, Laborde F, Leon MB. Percutaneous transcatheter implantation of an aortic valve prosthesis for calcific aortic stenosis: first human case description. Circulation 2002; 106: 3006-3008.
4. Elmariah S, MD, Palacios IF, McAndrew T, Hueter I, Inglessis I, Baker JN, Kodali S, Leon MB, Svensson L, Pibarot P, DVM, Douglas PS, Fearon WF, Kirtane AJ, Maniar HS, Passeri JJ; PARTNER Investigators. Outcomes of Transcatheter and Surgical Aortic Valve Replacement in High-Risk Patients with Aortic Stenosis and Left Ventricular Dysfunction. Results From the Placement of Aortic Transcatheter Valves (PARTNER) Trial (Cohort A). Circ Cardiovasc Interv 2013; 6: 604-614.

5. Svensson LG, Tuzcu M, Kapadia S, Blackstone EH, Roselli EE, Gillinov AM, Sabik JS, Lytle BW. A comprehensive review of the PARTNER trial. J Thorac Cardiovasc Surg 2013; 145: S11-S16.

6. Elmaraezy A, Ismail A, Abushouk Al, Eltoomy M, Saad S, Negida A, Abdelaty OM, Abdallah AR, Aboelfotoh AM, Hassan HM, Elmaraezy AG, Morsi M, Althaher F, Althaher M, AlSafadi AM. Efficacy and safety of transcatheter aortic valve replacement in aortic stenosis patients at low to moderate surgical risk: a comprehensive meta-analysis. BMC Cardiovascular Disorders 2017; 17: 234.

7. Enezate TH, Kumar A, Fadel MA, Patel M, Al Dadah A, Omran J. Transcatheter versus surgical aortic valve replacement in patients with non-high surgical risk severe aortic stenosis: a systematic review. Cardiovasc Revasc Med 2017; 18: S40-S48.

8. Spadaccio C, Nappi F, Sablayrolles JL, Sutherland FWH. TAVR vs. SAVR: rising expectations and changing indications for surgery in response to PARTNER II. Semin Thoracic Surg 2017; 29: 8-11.

9. Patel PA, Ackermann AM, Augoustides JG, Ender J, Gutsche JT, Giri J, Vallabhajosyula P, Desai ND, Kostibas M, Brady MB, Eoh EJ, Gaca JG, Thompson A, Michael Fitzsimons MG. Anesthetic evolution in transcatheter aortic valve replacement: expert perspectives from high-volume academic centers in Europe and the United States. J Cardiothorac Vasc Anesth 2017; 31: 777-790.

10. D’Errigo P, Ranucci M, Covello RM, Biancari F, Rosato S, Barbanti M, Onorati F, Tamburino C, Santoro G, Grossi C, Santini F, Bontempi K, Fusco D, Seccareccia F; the OBSERVANT Research Group. Outcome after general anesthesia versus monitored anesthesia care in transfemoral transcatheter aortic valve replacement. J Cardiothorac Vasc Anesth 2016; 30: 1238-1243.

11. Toppen W, Johansen D, Sareh S, Fernandez J, Satou N, Patel KD, Kwon M, Suh W, Aksoy O, Shemin RJ, Benharash P. Improved costs and outcomes with conscious sedation vs general anesthesia in TAVR patients: time to wake up? PLoS One 2017; 12: e0173777.

12. Palermo CH, Degnan M, Candiotti M, Salerno T, deMarchena E, RodriguezBlanco Y. Monitored anesthesia care versus general anesthesia: experience with the Medtronic CoreValve. J Cardiothorac Vasc Anesthesia 2016; 30: 1234-1237.

13. Mayr NP, Wiesner G, Husser O, Joner M, Michel J, Knorr J, Pellegrini C, Bleiziffer S, Schunkert H, Lange R, Tassani-Prell P. Critical adverse events during transfemoral TAVR in conscious sedation. Is an anesthesiologic support mandatory? Cardiovasc Revasc Med 2018; 19: S41-S46.

14. Kimmel J, Potosky R, Williams MR, Glading M, Neuburger PJ, Roberts JD, Feider A. Conversion from monitored anesthesia care to general anesthesia for transcatheter aortic valve replacement. J Cardiothorac Vasc Anesthesia 2018; 32: 1032-1040.

15. Neuburger PJ, Patel PA. Anesthetic techniques in transcatheter aortic valve replacement and the evolving role of the anesthesiologist. J Cardiothorac Vasc Anesth 2017; 31: 2175-2182.

16. Patel PA, Neuburger PJ. Postoperative delirium in transcatheter aortic valve replacement: future steps to make a meaningful attempt at prevention. J Cardiothorac Vasc Anesth 2017; 31: 1985-1987.

17. Soundhar A, Udesh R, Mehta A, Schnider J, Jeevanantham V, Gleason T, Thirumala PD. Delirium following transcatheter aortic valve replacement: national inpatient sample analysis. J Cardiothorac Vasc Anesth 2017; 31: 19771984.

18. Mittnacht AJ, Shariat A, Weiner MM, Malhotra A, Miller MA, Mahajan A, Bhatt HV. Regional techniques for cardiac and cardiac-related procedures. J Cardiothorac Vasc Anesth 2019; 33: 532-546.

19. Mayr NP, Michel J, Bleiziffer S, Tassani P, Martin K. Sedation or general anesthesia for transcatheter aortic valve implantation (TAVI). J Thorac Dis 2015; 7: 1518-1526.

20. Sola M, Ramm CJ, Kolarczyk LM, Teeter EG, Yeung M, Caranasos TG, Vavalle JP. Application of multidisciplinary enhanced Recovery after surgery pathway to improve patient outcomes after transcatheter Aortic Valve Implantation. Am J Cardiol 2016; 118: 418-423. 